\title{
Multiparametric Linear Complementarity Problems
}

\author{
Colin N. Jones and Manfred Morrari \\ Automatic Control Laboratory \\ Swiss Federal Institute of Technology \\ Physikstrasse 3, CH-8092 Zurich \\ Switzerland \\ \{jones\}\{morari\}@control.ee.ethz.ch
}

\begin{abstract}
The linear complementarity problem (LCP) is a general problem that unifies linear and quadratic programs and bimatrix games. In this paper, we present an efficient algorithm for the solution to multiparametric linear complementarity problems (pLCPs) that are defined by positive semi-definite matrices. This class of problems includes the multiparametric linear ( $p L P$ ) and semi-definite quadratic programs ( $p Q P$ ), where parameters are allowed to appear linearly in the cost and the right hand side of the constraints. We demonstrate that the proposed algorithm is equal in efficiency to the best of current pLP and pQP solvers for all problems that they can solve, and yet extends to a much larger class.
\end{abstract}

\section{INTRODUCTION}

It is standard practice to implement a model predictive controller (MPC) by solving on-line an optimisation problem where the decision variables provide the control action. In recent years, it has become well-known that the optimal input for a large class of systems is a piecewise affine function (PWA) defined over a polyhedral partition of the feasible states. By pre-computing this PWA function off-line, the on-line calculation of the control input then becomes one of evaluating the PWA function at the current measured state, which allows for significant improvements in sampling speed [1].

If the system is linear, the constraints polyhedral and the cost linear or quadratic, then the optimisation problem to be solved is a linear or quadratic program. Pre-computation of the PWA control law then requires the solution of a (multi) parametric linear (pLP) or quadratic program (pQP) [1]-[3]. These algorithms have a wider use in control, such as for constrained control allocation [4] for solving sub-problems in nonlinear optimisation [5] and for calculating penalty weights in soft-constrained linear MPC [6].

A large number of algorithms for pQPs [2], [3], [7]-[11] and pLPs [10], [12], [13] have been published in the control literature in the past few years. In [3], [12], [14] all optimal bases are enumerated with an algorithm based on the simplex approach. A geometric approach has been proposed [10] that directly explores the set of feasible parameters in a recursive manner, subdividing the feasible parameter space into socalled critical regions, in which the set of active constraints at the optimiser does not change. This method can introduce a large number of artificial cuts in the parameter space and an extension of this algorithm in [7], [11] addresses this problem by enumerating the regions in a non-recursive manner by stepping a sufficiently small distance over the facets of each region to find a point in a neighbouring region. In [8], [9] the algorithm for pQPs was again improved and it was shown that the adjacent critical region can be determined by inspection if it satisfies fairly strict nondegeneracy assumptions.

The algorithms [2], [7]-[11] can be applied only to strictly convex pQPs and they implicitly make the assumption that the intersection of two polyhedral critical regions is a face of each. However, it was shown in [15], [16] that this property does not hold either for strictly, or non-strictly convex pQPs or $\mathrm{pLPs} / \mathrm{pQPs}$ with parameterised costs and constraints, although it does hold for non-degenerate pLPs [13]. As a result, the algorithms [2], [7]-[11] cannot be guaranteed to return the correct solution in general. The paper [16] addressed this issue by combining the approach in [2] with that in [8], although some of the efficiency of [8] is lost.

In this paper, we present a new method based on [17] for computing the solution to a multi-parametric linear complementarity problem (pLCP), which is defined by a positive semi-definite matrix. Linear complementarity problems are considered fundamental and have been extensively studied in various disciplines as they unify linear, quadratic and bimatrix games. Specifically, the approach presented here provides the solution to linear and semi-definite quadratic multi-parametric programs in which parameters can appear linearly in both the cost and the right hand side of the constraints. Furthermore, the proposed approach is numerically robust, and is equal or superior in efficiency to [7], [11] for all problems that can be solved using those approaches. In the cases of non-degeneracy in pQPs, the proposed method reduces to [8], [9] and extends these ideas naturally to a much larger class of problems, whether degenerate or not.

The remainder of this paper is organised as follows. Section II provides the necessary background on LCPs and pLCPs. The proposed solution method is given in Section III, complexity analysis in Section IV and conclusions in Section V.

\section{NOTATION}

If $A \in \mathbb{R}^{m \times n}$ and $I \subseteq\{1, \ldots, n\}$, then $A_{*, I} \in \mathbb{R}^{m \times|I|}$ is the matrix formed by the columns of $A$ indexed by $I$. If $c \in \mathbb{R}^{n}$ is a vector then $c_{I}$ is the vector formed by the elements of $c$ in $I$. If $R \subseteq\{1, \ldots, m\}$ then we will use the 
notation $A_{R, *} \in \mathbb{R}^{|R| \times n}$ to be the matrix formed by the rows of $A$ indexed by $R$.

The set of affine combinations of points in a set $S \in \mathbb{R}^{n}$ is called the affine hull of $S$ and is denoted aff $(S)$. The dimension $\operatorname{dim}(S)$ of a set $S \subseteq \mathbb{R}^{n}$ is the dimension of $\operatorname{aff}(S)$. If $\operatorname{dim}(S)$ is equal to $n$, then the set is called full-dimensional. The closure of $S$ is denoted $\bar{S}$ and the relative interior is given by $\operatorname{relint}(S)\{x \in S \mid B(x, r) \cap \operatorname{aff}(S) \subseteq S$, for some $r>0\}$, where the ball $B(x, r) \triangleq\{y \mid\|x-y\|<r\}$ and $\|\cdot\|$ is any norm.

A polyhedron is the intersection of a finite number of closed halfspaces and a polytope is a bounded polyhedron. If $P=\{x \mid A x \leq b\}$ is a polyhedron and $H=\left\{x \mid a^{T} x \leq b\right\}$ is a halfspace such that $P \subseteq H$, then $P \cap\left\{x \mid a^{T} x=b\right\}$ is a face of $P$. The inequality $\left\{x \mid A_{i, *} x \leq b_{i}\right\}$ is called redundant if $P=\left\{x \mid A_{\{1, \ldots, n\} \backslash\{i\}, *} x \leq b_{\{1, \ldots, n\} \backslash\{i\}}\right\}$ and irredundant otherwise. If the dimension of the polytope is $d$, then the zero- and $(d-1)$-dimensional faces are called the vertices and the facets respectively.

\section{PRELIMINARIES}

A feasible solution to the following linear complementarity problem (LCP), denoted $(q, M)$, is a set of positive vectors $w$ and $z$ satisfying:

$$
w-M z=q, \quad w \geq 0, \quad z \geq 0, \quad w^{T} z=0,
$$

where the square matrix $M \in \mathbb{R}^{n \times n}$ and the vector $q \in \mathbb{R}^{n}$ are the problem data. The LCP is called feasible if there exist vectors $w$ and $z$ that satisfy (1).

Consider the following system of linear equality constraints in positive variables:

$$
A x=q, \quad x \geq 0,
$$

where $A \triangleq\left[\begin{array}{ll}I & -M\end{array}\right]$ and $x \triangleq\left[\begin{array}{ll}w^{T} & z^{T}\end{array}\right]^{T}$. Any set $B \subset\{1, \ldots, 2 n\}$ such that $|B|=n$ and $\operatorname{rank} A_{*, B}=n$ is called a basis and we write $N=\{1, \ldots, n\} \backslash B$ for its complement and call $x_{B}$ and $x_{N}$ the basic and non-basic variables respectively. A basis is called complementary if $i \in$ $B$ implies $i+n \notin B$ and $i-n \notin B$. Note that complementary bases are exactly those bases that satisfy the complementarity condition $w^{T} z=0$.

Every basis $B$ defines a solution to the linear equations in (2) or equivalently (1), which is given by restricting the non-basic constraints to zero

$$
x_{B}=A_{*, B}^{-1} q, \quad x_{N}=0 .
$$

A basis is called feasible if the resulting solution also satisfies the positivity constraints in (2): $A_{*, B}^{-1} q \geq 0$.

Clearly, a basis gives a feasible solution for the LCP $(q, M)$ if only if it is a complementary and feasible basis. Furthermore, every feasible solution to the LCP $(q, M)$ can be described by such a basis [18].

\section{A. Lexicographic Perturbation}

A basis $B$ is called degenerate if at least one component in the vector $A_{*, B}^{-1} q$ is zero, and non-degenerate otherwise. If a complementary feasible basis is degenerate then there exists more than one feasible basis for the given LCP. This non-uniqueness can cause several problems for parametric algorithms [13], and so in this paper we will remove the issue of degeneracy through the use of a lexicographic perturbation.

Definition 1 (Lexico-positive): A vector $a \in \mathbb{R}^{r}$ is said to be lexico-positive, denoted by $a \succeq 0$, if the first nonzero component in $a$ is strictly positive. Given two vectors $x, y \in$ $\mathbb{R}^{r}, x \succeq y$ if and only if $x-y \succeq 0$. The lexico-minimum of a set of vectors $\left\{a^{1}, \ldots, a^{m}\right\} \subset \mathbb{R}^{r}$ is the vector $a^{j}$ satisfying the property that $a^{i} \succeq a^{j}$ for each $i=1$ to $m$. A matrix is called lexico-positive if all rows of the matrix are lexico-positive.

Let $\epsilon \in \mathbb{R}^{n}$ be the vector $\left(\epsilon_{0}, \epsilon_{0}^{2}, \ldots, \epsilon_{0}^{n}\right)$, where $\epsilon_{0} \in \mathbb{R}$ is strictly positive. Consider the perturbed system of equalities in positive variables:

$$
A x=q+\epsilon, \quad x \geq 0 .
$$

If $B$ is a basis for (4) and $i \in B$, the the basic variables $x_{B}$ are given by:

$$
x_{i}=\beta_{B(i), *} q+\beta_{B(i), 1} \epsilon_{0}+\beta_{B(i), 2} \epsilon_{0}^{2}+\cdots+\beta_{B(i), n} \epsilon_{0}^{n},
$$

where $\beta \triangleq A_{*, B}^{-1}$ and $\beta_{B(i), *}$ is the row of $\beta$ associated to the variable $i$ (i.e. $i$ is the $B(i)^{\text {th }}$ element in $B$ ). One can see that the basis is feasible for sufficiently small $\epsilon_{0}$ if and only if the matrix $A_{B, *}^{-1}\left[\begin{array}{cc}q & I\end{array}\right]$ is lexico-positive; such a basis is called lexico-feasible.

We now state the main result of lexicographic perturbation:

Theorem 1 (Lexicographic Perturbation [19]): If an LCP $(q, M)$ is feasible, then there exists an $\epsilon_{1}>0$ such that for all $0<\epsilon_{0}<\epsilon_{1}$ the lexicographically perturbed LCP $(q+\epsilon, M)$ has a unique copmlementary feasible basis, where $\epsilon \triangleq\left(\epsilon_{0}, \epsilon_{0}^{2}, \ldots, \epsilon_{0}^{n}\right)$.

Remark 1: Note that $\epsilon$ is a symbolic perturbation. Only the effect of $\epsilon$ on the problem is considered and $\epsilon$ is never assigned a real value. As a result, there are no numerical concerns resulting from taking $\epsilon$ to be too small or too large a value.

\section{B. Parametric $L C P$}

The problem that we consider in this paper is the parametric linear complementarity problem, or $\mathrm{pLCP}(q+Q \theta+\epsilon, M)$ :

$$
w-M z=q+Q \theta+\epsilon, \quad w \geq 0, z \geq 0, \quad w^{T} z=0,
$$

where $Q \in \mathbb{R}^{n \times d}$ is a real matrix of rank $d, \epsilon$ is a lexicographic perturbation and $\theta \in \Theta \subseteq \mathbb{R}^{d}$ is the parameter. We make the following standing assumptions:

Assumption 1: The feasible set $\Theta$ is a full-dimensional polyhedron.

Assumption 2: There exists a finite feasible solution to the LCP $(q+Q \theta, M)$ for every $\theta \in \Theta$. 
Assumption 3: The matrix $M$ is positive semi-definite.

Definition 2 (Critical Region): If $B$ is a complementary feasible basis of the pLCP $(q+Q \theta+\epsilon, M)$, then the critical region $\mathscr{R}_{B}$ is defined as the set of all parameters $\theta_{\circ} \in \Theta$ such that $B$ is feasible for the LCP $\left(q+Q \theta_{0}+\epsilon, M\right)^{1}$.

From (3) and (5), it can be seen that the critical region $\mathscr{R}_{B}$ is the set

$$
\begin{aligned}
\mathscr{R}_{B} & =\{\theta \mid \beta(Q \theta+q+\epsilon) \geq 0\} \\
& =\left\{\theta \mid \beta\left[\begin{array}{ll}
Q \theta+q & I
\end{array}\right] \succeq 0\right\},
\end{aligned}
$$

where $A \triangleq\left[\begin{array}{ll}I & -M\end{array}\right], \beta \triangleq A_{*, B}^{-1}$ and $\succeq$ is taken rowwise. Since the perturbation $\epsilon$ is taken arbitrarily small, the closure of the critical region is the polyhedron

$$
\overline{\mathscr{R}}_{B}=\{\theta \mid \beta(Q \theta+q) \geq 0\} .
$$

Theorem 2: Let $\left\{\mathscr{R}_{B_{0}}, \ldots, \mathscr{R}_{B_{N}}\right\}$ be the set of all fulldimensional critical regions, then:

1) $\mathscr{R}_{B_{i}} \cap \mathscr{R}_{B_{j}}=\emptyset$ for all $i \neq j$

2) $\cup_{i=0}^{N} \overline{\mathscr{R}}_{B_{i}}=\Theta$

Proof: The first property follows directly from Theorem 1. Since every lexico-perturbed LCP has a unique feasible basis, every parameter $\theta$ must be in exactly one critical region.

The second property follows from the assumption that there exists a feasible solution for every value of the parameter in the set $\Theta$.

The goal is to compute a feasible basis for each value of the parameter in the set $\theta \in \Theta$. Since the pLCP is lexicographically perturbed a critical region $\mathscr{R}_{B}$ may or may not include its boundary. From a control point of view, the goal in solving a pLCP is to define a mapping from the measured state (the parameter) to the input (the variable $x$ ). Since the measurement of the state is never exact, we make the standard assumption that it will never lie in the relative interior of a critical region that is not full-dimensional and therefore enumerate only the full-dimensional regions.

\section{Parametric Quadratic Programming}

The main motivation for considering pLCPs from a control viewpoint is that all parametric linear and quadratic programs ( $\mathrm{pLPs}$ and pQPs) with positive semi-definite costs can be posed as pLCPs. This class of problems includes both parametric linear and quadratic programs in which both the cost and the right hand side of the constraints are parameterised.

\footnotetext{
${ }^{1}$ Note that this definition differs slightly from that generally given in the literature, in which the set of all active constraints is taken to define a critical region. However, as our problem has been lexicographically perturbed it is never degenerate and therefore these two definitions are equivalent.
}

Consider the following parameterised quadratic program ${ }^{2}$ :

$$
\begin{gathered}
\underset{u}{\operatorname{minimise}} \frac{1}{2} u^{T} D u+(E \theta+c)^{T} u \\
\text { subject to } A u \geq F \theta+b \\
u \geq 0
\end{gathered}
$$

where $D \in \mathbb{R}^{l \times l}, E \in \mathbb{R}^{l \times d}, c \in \mathbb{R}^{l}, A \in \mathbb{R}^{m \times l}, F \in \mathbb{R}^{m \times d}$ and $b \in \mathbb{R}^{m}$. The Karush-Kuhn-Tucker (KKT) optimality conditions for (9) are:

$$
\begin{gathered}
D u+E \theta+c-A^{T} \lambda-\nu=0 \\
\lambda^{T}(A u-F \theta-b)=0, \quad \nu^{T} u=0 \\
A u \geq F \theta+b, \quad u \geq 0
\end{gathered}
$$

Defining the slack variable $\sigma \triangleq A u-F \theta-b$ allows the KKT conditions (10) to be written as the following pLCP:

$$
\begin{gathered}
\left(\begin{array}{l}
\nu \\
\sigma
\end{array}\right)-\left[\begin{array}{cc}
D & -A^{T} \\
A & 0
\end{array}\right]\left(\begin{array}{l}
u \\
\lambda
\end{array}\right)=\left[\begin{array}{r}
E \\
-F
\end{array}\right] \theta+\left[\begin{array}{r}
c \\
-b
\end{array}\right] \\
\left(\begin{array}{l}
\nu \\
\sigma
\end{array}\right)^{T}\left(\begin{array}{l}
u \\
\lambda
\end{array}\right)=0, \quad \nu, \sigma, u, \lambda \geq 0
\end{gathered}
$$

Note that the matrix $M=\left[\begin{array}{cc}D & -A^{T} \\ A & 0\end{array}\right]$ is positive semidefinite and Assumption 3 is satisfied if $D$ is also positive semi-definite.

\section{REGION ENUMERATION}

The goal of the pLCP algorithm is to identify all lexicofeasible bases that define full-dimensional critical regions.

Definition 3 (Adjacent Regions [16]): Two

fulldimensional critical regions $\mathscr{R}_{A}$ and $\mathscr{R}_{B}$ are said to be adjacent if $\operatorname{dim}\left(\overline{\mathscr{R}}_{A} \cap \overline{\mathscr{R}}_{B}\right)=d-1$.

The algorithm proposed for this enumeration is similar to the geometric approach in [13] for solving pLPs and [7], [11] for pQPs. The exploration begins from a single fulldimensional critical region. Each of the facets of the closure of the region are considered in turn and all adjacent critical regions whose closures intersect the facet are enumerated. In turn each of these adjacent critical regions are considered and so on, until there are no more regions to be discovered. The algorithm is outlined below as Algorithm 1.

The following theorem, from [16] proves that this procedure will enumerate all full-dimensional critical regions.

Theorem 3 (Correctness of Algorithm [16]): If $\left\{\mathscr{R}_{B_{0}}, \ldots, \mathscr{R}_{B_{N}}\right\}$ is the set of full-dimensional critical regions returned by Algorithm 1, then $\cup_{i=0}^{N} \overline{\mathscr{R}}_{B_{i}}=\Theta$.

There are two operations that must be detailed for this approach: computation of the facets of the closure of a critical region, and computation of the adjacent regions containing a given facet. The following two sections cover these procedures.

${ }^{2}$ Note that the standard parametric quadratic program resulting from control problems does not have positivity constraints on the variables $u$. All parametric quadratic programs can be converted to the form (9) through a simple change of variables. 


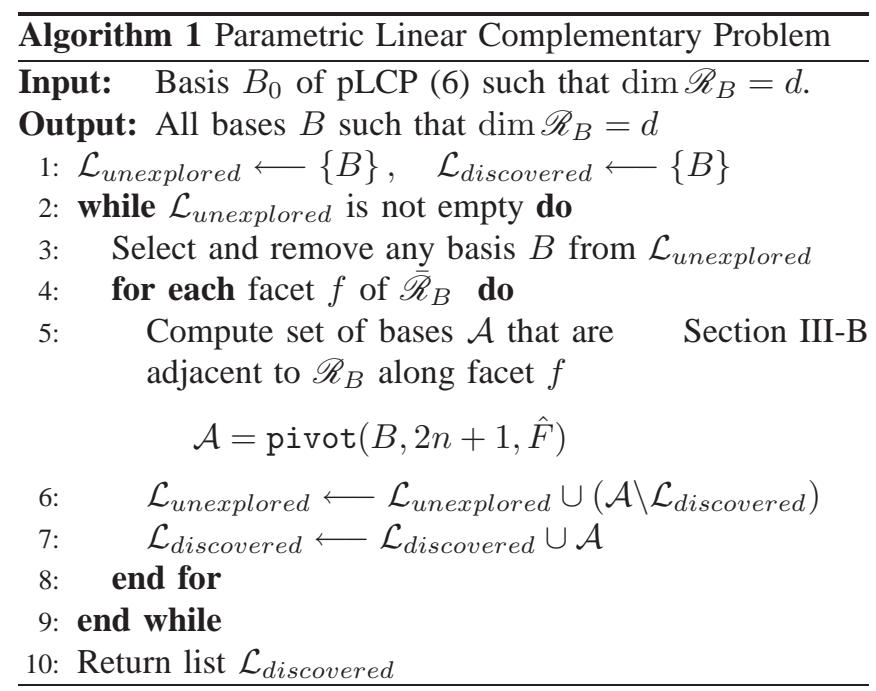

\section{A. Facet Calculation}

Consider the closure of the full-dimensional critical region $\mathscr{R}_{B}$, which is defined by a given basis $B$ :

$$
\overline{\mathscr{R}}_{B}=\{\theta \mid \beta(Q \theta+q) \geq 0\},
$$

where $\beta \triangleq A_{*, B}^{-1}$. The facets of $\overline{\mathscr{R}}_{B}$ are given by the irredundant inequalities of (11). Testing if an inequality is redundant requires a single linear program of dimension $d$. This is a standard redundancy elimination operation, and the reader is reffered to [20] for computational details.

\section{B. Adjacent Region Computation}

Given a full-dimensional critical region $\mathscr{R}_{B}$ and a hyperplane $f \triangleq\left\{\theta \mid \gamma^{T} \theta+b=0\right\}$, such that $f \cap \overline{\mathscr{R}}_{B}$ is a facet of the closure, the goal is to compute all adjacent critical regions $\mathscr{R}_{B^{\prime}}$ such that $\overline{\mathscr{R}}_{B} \cap \overline{\mathscr{R}}_{B^{\prime}} \subseteq f$ and $\operatorname{dim}\left(\overline{\mathscr{R}}_{B} \cap \overline{\mathscr{R}}_{B^{\prime}}\right)=d-1$.

If $\theta_{0}$ is a point in the relative interior of the facet $f \cap \overline{\mathscr{R}}_{B}$, then the goal is to compute the feasible basis for the pLCP at the point $\theta=\theta_{0}+\gamma \alpha$, which is just outside the critical region for some strictly positive $\alpha$. Note that we have assumed $\gamma$ to be facing outward from the facet and for convenience we also assume that $\|\gamma\|_{2}=1$.

In order to simplify the computations, we begin by rewriting the hyperplane $f$ as:

$$
f=\left\{\theta \mid \exists \theta_{f} \in \mathbb{R}^{d-1}, \theta=N \theta_{f}-\gamma b\right\},
$$

where $N \in \mathbb{R}^{d \times d-1}$ is a matrix whose columns span the left-nullspace of $\gamma$. Further, we define the set

$$
\hat{F} \triangleq\left\{\theta_{f} \in \mathbb{R}^{d-1} \mid N \theta_{f}-\gamma b \in f \cap \overline{\mathscr{R}}_{B} \cap \Theta\right\}
$$

The problem to be solved can now be written as the pLCP:

$$
\begin{array}{ll}
\left(q-Q \gamma b+Q N \theta_{f}+Q \gamma \alpha+\epsilon, M\right), & \alpha>0, \theta_{f} \in \hat{F} \\
\left(\hat{q}+\hat{Q} \theta_{f}-\hat{\gamma} \alpha+\epsilon, M\right), & \alpha>0, \theta_{f} \in \hat{F}
\end{array}
$$

where $\hat{q} \triangleq q-Q \gamma b, \hat{Q} \triangleq Q N$ and $\hat{\gamma} \triangleq-Q \gamma$.

We now describe an algorithm similar to that given in [18] for one-dimensional parametric linear complementarity problems in the parameter $\alpha$, where augmentations are made in order to handle the parametric nature of $\theta_{f}$. The algorithm proceeds through pivoting operations, beginning at the basis $B$. At each stage, an entering variable is chosen from the non-basic constraints and is increased until one of the basic variables decreases to zero. At this point this basic variable, called the leaving variable, is removed from the basis, and the entering variable is brought into it. This procedure maintains the feasibility of the new basis, continuing until we arrive at a basis $B^{\prime}$ that remains complementary and feasible for a strictly positive value of $\alpha$.

Consider the following linear equalities in positive variables:

$$
\hat{A} \hat{x}=\hat{q}+\hat{Q} \theta_{f}, \quad \hat{x} \geq 0
$$

where $\hat{A} \triangleq\left[\begin{array}{lll}-I & M & \hat{\gamma}\end{array}\right] \in \mathbb{R}^{n \times 2 n+1}$ is a matrix and $\hat{x} \triangleq(x, \alpha)^{T} \in \mathbb{R}^{2 n+1}$ is a positive vector.

Definition 4 (ACLFB [18]): A basis $B$ of (12) is an almost complementary lexico-feasible basis (ACFLB) if it

1) is lexico-feasible

2) contains at most one element from each pair of complementary variables $\left\{w_{i}, z_{i}\right\}$

3) contains exactly one basic variable from each of $(n-1)$ complementary pairs of variables, and the remaining pair is non-basic

1) Pivoting: The pivoting function takes as input an entering variable $\hat{x}_{e}, e \in N$, and an ACLFB $B$, which is assumed to contain the variable $\alpha$ and to be lexico-feasible for all $\theta_{f}$ in the set $\hat{F}$ :

$$
\hat{x}_{B}=\beta\left(\hat{q}+\hat{Q} \theta_{f}+\epsilon\right) \geq 0,
$$

where $\beta \triangleq \hat{A}_{*, B}^{-1}$.

a) Leaving Variable: Consider now the effect of increasing the variable $\hat{x}_{e}$ while maintaining lexico-feasibility:

$$
\hat{x}_{B}=\beta\left(\hat{q}+\hat{Q} \theta_{f}+\epsilon\right)-\beta \hat{A}_{*, e} \hat{x}_{e} \geq 0
$$

As $\hat{x}_{e}$ increases, the first constraint to become active is clearly given by:

$$
l=\operatorname{lexmin}\left\{\frac{\beta_{B(i), *}\left[\begin{array}{cc}
\hat{q}+\hat{Q} \theta_{f} & I
\end{array}\right]}{\beta_{B(i), *} \hat{A}_{*, e}} \mid i \in \mathcal{P}\right\},
$$

where $\mathcal{P} \triangleq\left\{i \mid \beta_{B(i), *} \hat{A}_{*, e}>0\right\}$ and $\beta_{B(i), *}$ is the row of $\beta$ associated to the variable $i$ (i.e. $i$ is the $B(i)^{t h}$ element in $B)$. As the variable $\hat{x}_{l}$ is now equal to zero, it is removed from the basis and the entering variable $e$ is added to it and allowed to be positive. Recall that all non-basic variables are zero and only basic variables can be positive. Note that because the leaving variable is chosen in order to maintain lexico-feasibility, the new basis $B^{\prime}=B \cup\{e\} \backslash\{l\}$ will also be an ACLFB.

We now consider how (13) is computed. We define the set $\mathcal{Z} \triangleq\left\{i \mid \beta_{B(i), *}\left[\begin{array}{cc}\hat{q} & \hat{Q}\end{array}\right]=0\right\}$ and differentiate two $\operatorname{cases}^{3}$ :

\footnotetext{
${ }^{3}$ The set $\mathcal{Z}$ contains all of the inequalities of the closure of the critical region that are equal to the facet hyperplane $f$, or are active everywhere in $\mathscr{R}_{B}$.
} 
Case $1 \mathcal{Z} \cap \mathcal{P} \neq \emptyset$

In this case, one can see that the minimum in (13) will be given by:

$$
l=\operatorname{lexmin}\left\{\frac{\beta_{i, *}}{\beta_{i, *} \hat{A}_{*, e}} \mid i \in \mathcal{P} \cap \mathcal{Z}\right\},
$$

Notice that (14) is independent of the parameter $\theta_{f}$.

Remark 2: Note that the minimum in (14) is always unique since the choice could only be non-unique if $\beta$ were not invertible.

Remark 3: If every pivot of a problem is in this case then the adjacent region is independent of the point on the facet $\theta_{f}$ and therefore satisfies the so-called facet-to-facet property [16]

Case $2 \mathcal{Z} \cap \mathcal{P}=\emptyset$

In this case, the leaving variable will be a function of the parameter $\theta_{f}$. There may be more than one possible leaving variable and therefore more than one adjacent critical region along this facet.

Theorem 4: If $B$ is an ACLFB, $e$ is an entering variable and $\mathcal{Z} \cap \mathcal{P}=\emptyset$, then the basis $B^{\prime}=B \cup\{e\} \backslash\{l\}$ is an ACLFB and $\operatorname{dim}\left(\overline{\mathscr{R}}_{B} \cap \overline{\mathscr{R}}_{B^{\prime}}\right)=d-1$ if and only if

$$
\operatorname{dim}\left\{\theta_{f} \in \mathbb{R}^{d-1} \mid \Gamma\left(\hat{Q} \theta_{f}+\hat{q}\right) \geq 0\right\} \cap \hat{F}=d-1
$$

and

$$
\Gamma_{i}\left[\begin{array}{cc}
\hat{q} & I
\end{array}\right] \succeq 0 \quad \text { for all } i \in \mathcal{P} \text { such that } \quad \Gamma_{i} \hat{Q}=0
$$

where

$$
\Gamma_{i} \triangleq\left(\beta_{i, *} \hat{A}_{*, e}\right) \beta_{l, *}-\left(\beta_{l, *} \hat{A}_{*, e}\right) \beta_{i, *}, \quad \forall i \in \mathcal{P}
$$

Proof: The variable $\hat{x}_{l}, l \in \mathcal{P}$ can be the leaving variable if and only if there exists a $\theta_{f} \in \operatorname{int} \hat{F}$ such that $l$ is the minimum in (13). This condition can be posed as the following set of linear lexico-inequalities:

$$
\frac{\beta_{i, *}\left[\begin{array}{ll}
\hat{q}+\hat{Q} \theta_{f} & I
\end{array}\right]}{\beta_{i, *} \hat{A}_{*, e}} \succeq \frac{\beta_{l, *}\left[\begin{array}{ll}
\hat{q}+\hat{Q} \theta_{f} & I
\end{array}\right]}{\beta_{l, *} \hat{A}_{*, e}}, \quad \forall i \in \mathcal{P}
$$

Straightforward algebraic manipulation converts (16) to the two conditions given in the statement of the theorem.

Each parameter $\theta_{f}$ that satisfies the conditions of the theorem will be in the closures of both critical region $\mathscr{R}_{B}$ and $\mathscr{R}_{B^{\prime}}$. Therefore, their intersection is of dimension $d-1$ if and only if the polytope (15) is full-dimensional.

Remark 4: Note that a polytope can be tested for fulldimensionality through the use of a single linear program by computing the Chebyshev centre [21].

Remark 5: We consider only full-dimensional intersections in Theorem 4 because Theorem 3 demonstrates that this is sufficient to guarantee that all full-dimensional critical regions will be discovered.

Remark 6: Note that once a pivot has been made using Theorem 4 in which the value of $\theta_{f}$ determines the leaving variable, the constraints (15) must hold for all future pivots. This is ensured by setting $\hat{F} \leftarrow\left\{\theta_{f} \mid \Gamma\left(\hat{Q} \theta_{f}+\hat{q}\right) \geq 0\right\} \cap$ $\hat{F}$ for all subsequent pivots. b) Entering Variable: After a pivot is taken, the resulting basis is required to be an ACLFB. As a result, the next entering variable must be chosen to be the complement of the leaving variable. In other words if $x_{l}=w_{i}$, then the entering variable $x_{e}$ would be $z_{i}$, and vice versa.

c) Termination Conditions:

Theorem 5 (Optimal Termination Condition): If $B$ is an ACLFB, $2 n+1 \in B$ and $e$ is an entering variable, then the basis $B^{\prime}=B \cup\{e\} \backslash\{2 n+1\}$ is a complementary lexico-feasible basis such that $\operatorname{dim} \mathscr{R}_{B^{\prime}}=d$ and $\operatorname{dim}\left(\overline{\mathscr{R}}_{B} \cap \overline{\mathscr{R}}_{B^{\prime}}\right)=d-1$ if

1) $\mathcal{P} \cap \mathcal{Z}=\emptyset$

2) $2 n+1 \in \mathcal{P}$

Proof: The goal is to find a feasible basis for which $\alpha$ can be increased to a strictly positive value. If $x_{e}$ is the entering variable, then $\alpha$ is given by the equation:

$$
\alpha=\beta_{B(2 n+1), *}\left(\hat{q}+\hat{Q} \theta_{f}+\epsilon-\hat{A}_{*, e} \hat{x}_{e}\right),
$$

where $\beta \triangleq \hat{A}_{*, e}^{-1}$ and we recall that $\alpha=\hat{x}_{2 n+1}$. We note, however, that $B(2 n+1)$ is in the set $\mathcal{Z}$; $\beta_{B(2 n+1), *}\left[\begin{array}{ll}\hat{q} & \hat{Q} \theta_{f}\end{array}\right]=0$. This is because if $B(2 n+1)$ is not in the set $\mathcal{Z}$, then by definition there exists a $\theta_{f} \in \hat{F}$ such that $\alpha>0$.

Therefore, increasing the variable $x_{e}$ will only cause $\alpha$ to be a strictly positive value if $x_{e}$ itself becomes strictly positive and $\beta_{B(2 n+1), *} \hat{A}_{*, e}<0$, which are precisely the conditions of the theorem.

Theorem 5 provides the conditions under which the algorithm terminates with a list of adjacent critical regions. Note however, that if the set $\mathcal{P}=\emptyset$, then either the parameter $\alpha$ or another variable can be increased forever without the basis becoming infeasible. It follows that in this case the facet is on the boundary of the feasible region $\Theta$.

d) Initialisation: The algorithm begins by bringing $\alpha$ into the feasible complementary basis $B$ by calling the function pivot with $\alpha$ as the entering variable. The pivoting function will then continue recursively until one of the termination conditions in Section III-B.1.c is reached, at which point either a list of adjacent regions will have been computed, or it will have been discovered that the facet is on the boundary of the feasible region.

e) Correctness: The above procedure is a generalisation of the one-dimensional parametric LCP method presented in [17]. Given a basis $B^{\prime}$ that defines an adjacent region, the above procedure takes precisely the same pivots as that in [17] for a fixed $\theta_{f}$ in the relative interior of $\overline{\mathscr{R}}_{B} \cap \overline{\mathscr{R}}_{B^{\prime}}$. For a fixed value of $\theta_{f}$, the following theorem proves the correctness of the algorithm. It follows that the above method computes all adjacent full-dimensional bases.

Theorem 6 ([17]): Consider the parametric LCP $(q+$ $\gamma \alpha, M)$, where $M$ is positive semi-definite and $B$ is a feasible basis for $\alpha=0$. The algorithm discussed above finds a solution to this pLCP for a strictly positive $\alpha$ in a finite number of pivot steps. Also, the solution obtained is the unique solution of this parametric LCP. 


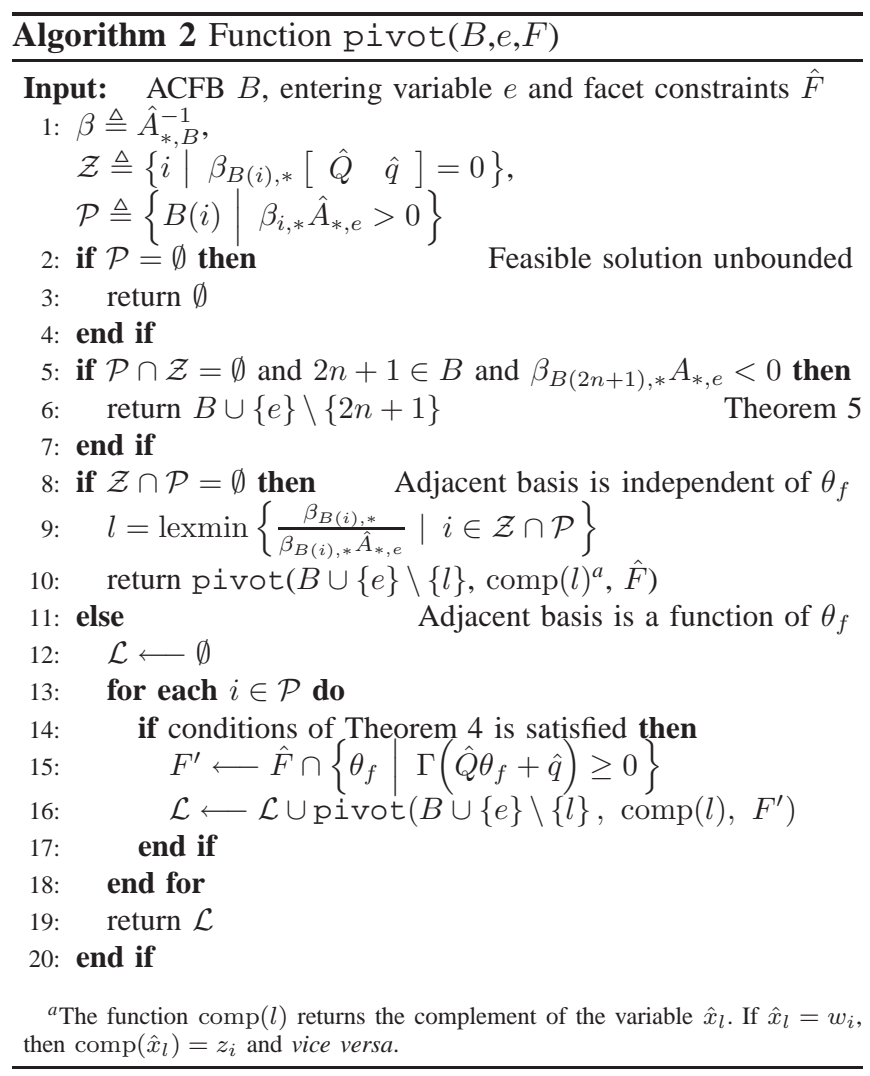

\section{COMPLEXity}

The above procedure is output sensitive, in that it computes the facets and calculates the adjacent neighbours of each full-dimensional critical region exactly once. We note that for every problem that the methods [7], [11], [13] can solve, the proposed approach will not execute a larger number of operations, since these problems will always fall into Case 1 in Section III-B.1. Furthermore, in every case that [8], [9] can determine the adjacent critical region by inspection, (13) will contain exactly one element and therefore one pivot will be made to find the adjacent region.

We therefore claim that the approach presented in this paper is computationally efficient as it is equal in complexity to the best known methods for all problems that they can solve, and yet extends to a much larger class of problems.

\section{CONCLUSIONS}

This paper has presented a new method for computing the solution to a multiparametric complementarity problem. The proposed approach is as efficient as the current best methods for computing multiparametric linear and quadratic programs, and yet can tackle a much larger class of problems.

Space limitations prevented the inclusion of any examples in this paper. However, code and examples are available as part of the Multiparametric Toolbox MPT [22].

\section{REFERENCES}

[1] A. Bemporad, F. Borrelli, and M. Morari, "Model predictive control based on linear programming - the explicit solution," IEEE Transactions on Automatic Control, vol. 47, no. 12, pp. 1974-1985, 2002.

[2] A. Bemporad, M. Morari, V. Dua, and E. Pistikopoulos, "The explicit linear quadratic regulator for constrained systems," Automatica, vol. 38, no. 1, pp. 3-20, Jan. 2002.

[3] M. M. Seron, G. C. Goodwin, and J. A. D. Doná, "Characterisation of receding horizon control for constrained linear systems," Asian Journal of Control, vol. 5, no. 2, pp. 271-286, 2003.

[4] T. A. Johansen, T. I. Fossen, and P. Tøndel, "Efficient optimal constrained control allocation via multi-parametric programming," $J$. Guidance, Control and Dynamics, 2003, submitted.

[5] T. A. Johansen, "On multi-parametric nonlinear programming and explicit nonlinear model predictive control," in Proc. 41st IEEE Conf. on Decision and Control, vol. 3, Las Vegas, NV, 2002, pp. 2768-2773.

[6] E. C. Kerrigan and J. Maciejowski, "Soft constraints and exact penalty functions in model predictive control," in Proc. UKACC International Conference (Control 2000), 2000.

[7] M. Baotić, "An efficient algorithm for multi-parametric quadratic programming," ETH Zürich, Institut für Automatik, Physikstrasse 3, CH-8092, Switzerland, Tech. Rep., 2002.

[8] P. Tøndel, T. Johansen, and A. Bemporad, "An algorithm for multiparametric quadratic programming and explicit MPC solutions," $A u-$ tomatica, pp. 489-497, 2003.

[9] _ - "Further results on multi-parametric quadratic programming," in 42nd IEEE Conf. on Decision and Control, Hawaii, 2003, pp. 3173 3178 .

[10] F. Borrelli, A. Bemporad, and M. Morari, "A Geometric Algorithm for Multi-Parametric Linear Programming," Journal of Optimization Theory and Applications, vol. 118, no. 3, pp. 515-540, September 2003.

[11] P. Grieder, F. Borrelli, F. Torrisi, and M. Morari, "Computation of the constrained infinite time linear quadratic regulator," Automatica, vol. 40, pp. 701-708, 2004.

[12] T. Gal, Postoptimal Analyses, Parametric Programming and Related Topics, $2^{\text {nd }}$ ed. Walter de Gruyter, 1995.

[13] C. Jones, E. Kerrigan, and J. Maciejowski, "Lexicographic perturbation for multiparametric linear programm ing with applications to control," Automatica, 2005, submitted.

[14] M. Schechter, "Polyhedral functions and multiparametric linear programming," Journal of Optimization Theory and Applications, vol. 53, no. 2, pp. 269-280, May 1987.

[15] M. Baric, M. Baotic, and M. Morari, "On-line Tuning of Controllers for Systems with Constraints," in IEEE Conference on Decision and Control, Sevilla, Spain, Dec. 2005, pp. 8288-8293.

[16] J. Spjøtvold, E. Kerrigan, C. Jones, T. Johansen, and P. Tøndel, "On the facet-to-facet property of convex parametric quadratic programs," Automatica, 2005, to appear.

[17] K. Murty, "On the parametric complementarity problem," in Engineer ing Summer Conference Notes, The University of Michigan, 1971.

[18] K. Murty and F. Yu, Linear Complementarity, Linear and Nonlinear Programming. Helderman-Verlag, 1988.

[19] K. Fukuda, H. Lüthi, and M. Namkiki, "The existence of a short sequence of admissible pivots to an optimal basis in LP and LCP," International Transactions of Operational Research, vol. 4, no. 4, pp. 273-384, 1997.

[20] K. Fukuda, "Frequently asked questions in polyhedral computation," 2004, http://www.ifor.math.ethz.ch/fukuda/polyfaq/polyfaq.html.

[21] S. Boyd and L. Vandenberghe, Convex Optimization. Cambridge University Press, 2004.

[22] M. Kvasnica, P. Grieder, and M. Baotić, "Multi-Parametric Toolbox (MPT)," 2004, http://control.ee.ethz.ch/ mpt/. 